\title{
Energetic Electron and Ion Beam Generation in Plasma Opening Switches
}

\author{
Yakov E. Krasik and Amit Weingarten
}

\begin{abstract}
In this paper, we present measurements of ion and electron flows in a nanosecond plasma opening switch (NPOS) and a microsecond plasma opening switch (MPOS), performed using charge collectors. In both experiments, an electron flow toward the anode, followed by an ion flow, were observed to propagate downstream toward the load side of the plasma during the plasma opening switch (POS) conduction. In the MPOS, ion acceleration was observed to propagate axially through the entire plasma. These results are in satisfactory agreement with the predictions of the electron magneto-hydrodynamics (EHMD) theory and the results of fluid and particle-in-cell (PIC) code simulations. At the beginning of the POS opening, a high-current density $\left(\approx 2 \mathrm{kA} / \mathrm{cm}^{2}\right)$ short-duration (10-30 ns) axial ion flow downstream toward the load was observed in both experiments, with an electron beam in front of it. These ions are accelerated at the load side of the plasma and are accompanied by comoving electrons. In the NPOS, the ion energy reaches $1.35 \mathrm{MeV}$, whereas in the MPOS, the ion energy does not exceed $100 \mathrm{keV}$. We suggest that in the NPOS the dominant mechanism for the axial ion acceleration is collective acceleration by the space charge of the electron beam, while in the MPOS, axial ion acceleration is probably governed by the Hall field in the current carrying plasma.
\end{abstract}

Index Terms-Acceleration, electron beams, Hall effect, inductive energy storage, ion beams, plasmas, power generation, pulse power system switches.

\section{INTRODUCTION}

D URING the last 20 years many experimental investigations were devoted to the plasma opening switch (POS) operated in nanosecond (NPOS) [1] and microsecond (MPOS) [2], [3] time scales. Such interest results from the POS potential as the fastest high-current, high-voltage interrupter in inductive energy storage systems and the rich physical phenomena taking place in the current carrying plasma.

Previous experimental investigations showed two main phenomena: a fast magnetic field penetration through the entire plasma [4], [5] and a fast increase of the switch impedance [1], [2]. The first phenomenon is explained within the framework

Manuscript received August 7, 1997; revised December 15, 1997. This work was supported by the Minerva Foundation, Munich, Germany, by the Office of Naval Research under Grant N00014-91-j-4104, and by the Israeli Academy of Science and Ministry of Absorption.

Ya. E. Krasik was with the Physics Department, The Weizmann Institute of Science, 76100 Rehovot, Israel. He is now with the Physics Department, Technion, 32000 Haifa, Israel.

A. Weingarten is with the Physics Department, The Weizmann Institute of Science, 76100 Rehovot, Israel.

Publisher Item Identifier S 0093-3813(98)02723-4. of the electron magneto-hydrodynamics (EMHD) theory based on the Hall effect [6]-[9]. Fast increase of the POS impedance is assumed to be associated with the anomalous fast magnetic field penetration [4] and the formation of a nonneutral layer [10] in the vicinity of the cathode or inside the plasma.

The main POS application is a fast transfer of inductively stored energy to a parallel load. Studying the energy losses during the POS operation that determine the maximum POS energy transfer efficiency is important. Direct measurements of current densities of electron and ion flows toward the POS electrodes and toward the load may improve the understanding of the physical phenomena and may lead to the improvement of the POS operation.

In previous investigations in both NPOS [11] and in MPOS [12], it was shown that during the opening, most of the energy lost by electron flow toward the anode is located at the load side of the plasma. Studies of the ion current toward the cathode during the NPOS and MPOS operation [13]-[15] show that the ion flow appears early in the current pulse at the generator side of the plasma and propagates toward the load side of the plasma. The maximum ion flux was measured during the opening along the entire POS length with the ions having energy similar to the induction POS voltage. The total energy carried by this ion flux can reach significant values of the inductively stored energy, up to $20 \%$ in an MPOS [16]. In our previous study of an NPOS with a positively charged central electrode, we have shown that during the opening, protons are accelerated at the load side of the plasma toward the short-circuit inductive load [16]. Also, there is evidence of the existence of energetic electron and ion flows downstream to the load during the opening of a MPOS [17].

In this paper we present measurements of ion and electron flows toward the anode and downstream toward the load during the operation of a coaxial NPOS (with the central electrode negatively charged) and a planar geometry MPOS. In Section II we describe the POS experimental setups, the plasma parameters, and the diagnostics used. The results of the collimated Faraday cup (CFC) measurements are presented in Section III and discussed in Section IV.

\section{THE EXPERIMENTAL SETUPS}

\section{A. The NPOS}

The NPOS experiment, shown in Fig. 1(a), consists of a coaxial anode and cathode with radii of 4.5 and $2 \mathrm{~cm}$, respectively. The NPOS is powered by a $300 \mathrm{kV}, 1 \Omega$, LC- 


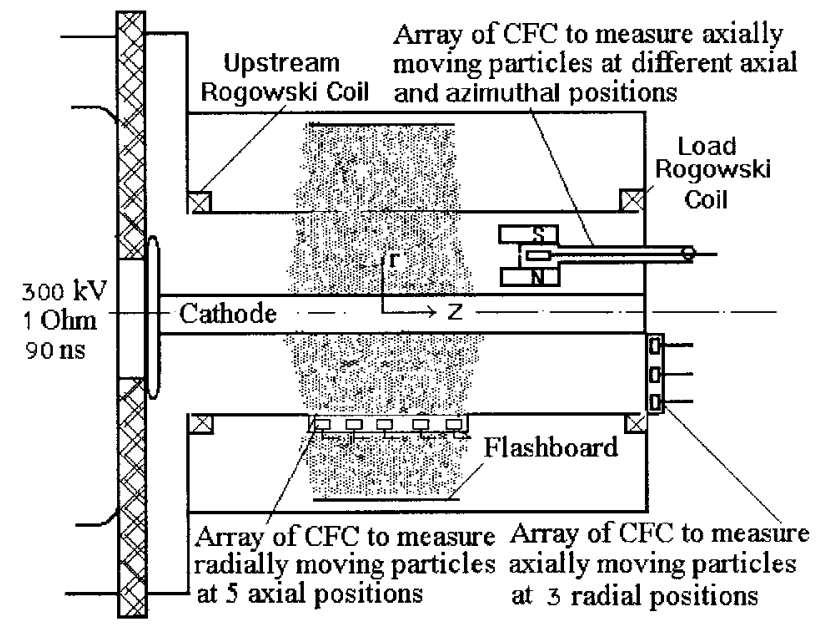

(a)

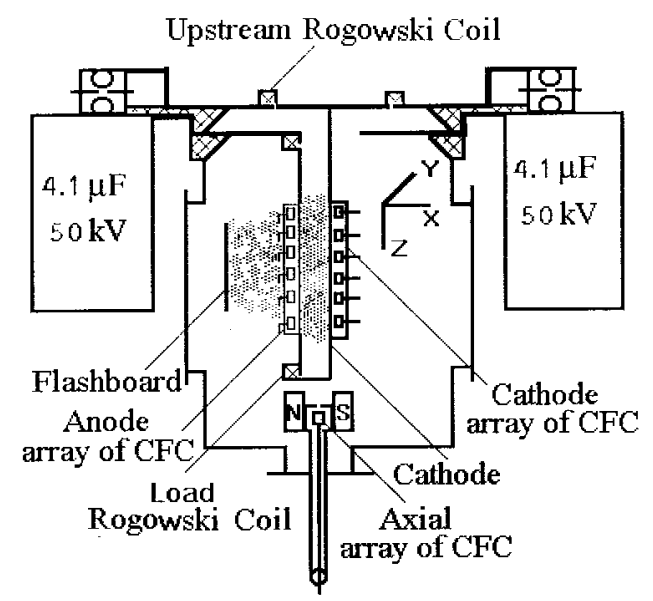

(b)

Fig. 1. The experimental setups of (a) the NPOS and (b) the MPOS.

water line generator producing a negative polarity current pulse: $I_{u}=200 \mathrm{kA}$ and $\tau_{1 / 4}=90 \mathrm{~ns}$. The NPOS upstream inductance is $L_{u}=90 \mathrm{nH}$ (length $30 \mathrm{~cm}$ ) and the load is a short-circuited coaxial line $L_{d}=20 \mathrm{nH}$ (length $12 \mathrm{~cm}$ ) having the same radii as the upstream section. Two self-integrated Rogowski coils are used to measure the upstream $\left(I_{u}\right)$ and the load $\left(I_{d}\right)$ currents.

For plasma prefilling, two 30-cm-long flashboard plasma sources are installed opposite each other at a radius of 11 $\mathrm{cm}$, outside the $75 \%$ transparent anode. Each flashboard is composed of four parallel chains. The power supply for the flashboard (capacitance of $0.9 \mu \mathrm{F}$, inductance of $300 \mathrm{nH}$, $\varphi \leq 32 \mathrm{kV}$ ) produces a $750 \mathrm{~ns}$ rise-time current pulse with amplitude $\leq 6 \mathrm{kA}$ through each discharge chain.

The electron density at the time the POS current is applied, determined from Stark broadening of $H_{\alpha}$ and $H_{\beta}$ line profiles, was found to be $2 \times 10^{14} \mathrm{~cm}^{-3}$. The average radial velocity of a hydrogen-carbon plasma determined by the time delay of the line emission was found to be $(4-7) \times 10^{6} \mathrm{~cm} / \mathrm{s}$. The axial length of the plasma prefilled region in the middle of the anode-cathode gap (where the density has dropped to half the density at the center) obtained by negatively biased CFC was found to be $\cong 7 \mathrm{~cm}$. In the following, "the generator side" and "the load side" of the plasma refers to the axial position where the density has dropped to half the density at the center, i.e., $\pm 3.5 \mathrm{~cm}$ from the POS region center.

The conduction time of the NPOS is controlled by the time delay between firing the flashboard power supply and the generator. The optimal NPOS opening time was found to be $\tau_{\text {op }}=(15 \pm 5)$ ns determined from the $10-90 \%$ rise-time of the load current that is $I_{d} \approx(150 \pm 20) \mathrm{kA}$. The conduction duration in this case is $\tau_{c} \approx 60-80$ ns without any measurable load current prepulse.

\section{B. The MPOS}

The MPOS experimental setup, shown in Fig. 1(b), is built of a low-inductance generator $\left(C_{g}=0.72 \mu \mathrm{F}, \varphi_{g} \leq 45\right.$ $\left.\mathrm{kV}, L_{g} \leq 25 \mathrm{nH}\right)$ which supplies a current pulse with an amplitude of $I_{u} \approx 230 \mathrm{kA}$ and a $750 \mathrm{~ns}$ rise-time when a short circuit is placed at the position of the plasma prefilled region. The MPOS consists of a strip line that has a $14-\mathrm{cm}$ width and a $3.6-\mathrm{cm}$ interelectrode gap. The upstream and the downstream sections of the strip line are 18 and $12 \mathrm{~cm}$ long, respectively, giving inductances of $L_{u}=60 \mathrm{nH}$ and $L_{d}=45$ $\mathrm{nH}$, respectively. The $I_{u}$ and the $I_{d}$ currents are measured by two self-integrated Rogowski coils.

Plasma injection into the MPOS interelectrode gap is implemented through anode slots by a flashboard plasma source placed $6 \mathrm{~cm}$ from the anode. The flashboard consists of eight parallel chains displaced $1 \mathrm{~cm}$ from one another. The power supply for this flashboard $\left(C_{g}=2.8 \mu \mathrm{F}, \varphi_{g} \leq 35 \mathrm{kV}\right)$ provides a current pulse with amplitude of $\approx 90 \mathrm{kA}$ and a $1.4 \mu$ s rise-time.

The axially averaged electron density and the average radial velocity of a hydrogen-carbon plasma determined by the same methods as in the NPOS experiment were found to be $n_{e} \approx$ $(8 \pm 2) \times 10^{14} \mathrm{~cm}^{-3}$ and $(6-8) \times 10^{6} \mathrm{~cm} / \mathrm{s}$, respectively. The axial length of the plasma prefilled region was determined to be $\sim 14 \mathrm{~cm}$, and as in the NPOS, "the generator side" and "the load side" of the plasma refer to the axial position where the density has dropped to half the density at the center $( \pm 7$ $\mathrm{cm}$ from center).

The MPOS conduction time, $\tau_{c}=(0.3-0.7) \mu \mathrm{s}$, is controlled by varying the delay between firing the flashboard and the generator. The best $\tau_{\mathrm{op}}=(45 \pm 5) \mathrm{ns}$ is achieved for $\tau_{c}=(400 \pm 50)$ ns. However, before the sharp opening of the MPOS, a slow rise of the load current starting $100 \mathrm{~ns}$ after the beginning of the upstream current is observed [see Fig. 7(a)]. This load current prepulse probably occurs because of edge effects of the strip line that allow partial current penetration at the edges of the strip line.

\section{The $C F C$}

The electron and ion emission from the plasma during POS operation were studied by several arrays of the magnetically insulated CFC's. Each CFC is made of brass, has a 0.4-cm outer diameter, and is placed inside an individual screen box. The distance of $0.2 \mathrm{~cm}$ between the cup and the collimating 
hole allows particles with a divergence angle of $\sim 90^{\circ}$ to be collected. Permanent magnets $(B=1 \mathrm{kG})$ placed above the CFC were used to cut off comoving electrons and to suppress secondary electron emission from the Faraday cup surface. The diameter of the collimating holes was chosen to satisfy the condition to suppress a polarization of the ion microbeam when it propagates in a cross magnetic field [18]: $r_{c} \leq 1.7 \cdot 10^{-4} E_{i} B / j_{i} \leq 0.025 \mathrm{~cm}$; here $B(T)$ is the cross magnetic field, $j_{i}\left(\mathrm{~A} / \mathrm{cm}^{2}\right)$ is the ion current density, $j_{i} \leq 1$ $\mathrm{kA} / \mathrm{cm}^{2}, E_{i}(\mathrm{eV})$ is the energy of ions, and $E_{i} \leq 1.5 \mathrm{MeV}$. The possible plasma prefilling of the CFC screen boxes was minimized by a $60 \%$ transparency stainless mesh placed 0.2 $\mathrm{cm}$ in front of the collimating holes.

In the NPOS experiment, the current density of the ion flow propagating in the axial direction toward the load was measured by an array of four axially movable CFC's, azimuthally separated by $90^{\circ}$ and placed at the middle of the interelectrode gap. The radial distribution of the axial ion beam current density was measured by an array of three magnetically insulated CFC's placed $10 \mathrm{~cm}$ from the load side of the plasma. To measure axial ion flow in the MPOS experiment, we used three movable arrays of magnetically insulated CFC's. Each array consists of four CFC's displaced $0.7 \mathrm{~cm}$ from each other that were placed across the interelectrode gap. The distance between the arrays in the " $Y$ " direction was $4.5 \mathrm{~cm}$.

We have verified that the CFC signals indeed result from charged particles by placing foils in front of the collimating holes. The ion axial energy was determined by time of flight (TOF) calculations and by placing aluminum foils and Kimfols of different thickness in front of the CFC holes. As we show in Section III, the ion energy in the MPOS was low, and the signals vanish even with the thinnest foil. Therefore, the velocity is determined only by TOF calculations. It is possible that in places where there is plasma in the vicinity of the CFC before the POS operation, the TOF calculation shows the "virtual" velocity of a current conducting region propagating through the plasma. Such a current channel would accelerate the ions to the CFC, but the velocity of the ions could be lower than the current channel propagation velocity. In this case, the TOF calculation gives an upper limit for the ion velocity. Nevertheless, in the regions where there is no plasma prior to the POS operation, the TOF calculations show the true ion velocity.

The TOF measurements were made by the CFC array when each cup was positioned at a different axial position. In a single generator shot, the maximum axial displacement between the four CFC's in the array was up to $8 \mathrm{~cm}$. Since an uncertainty in the TOF calculation may also result from mixing of ions with different energies accelerated at different times, drift tubes of 20 and $25 \mathrm{~cm}$ length for the NPOS and MPOS, respectively, were added beyond the short-circuit loads.

Measurements of electron and ion flow toward the anode were made by an array of six CFC's in the MPOS experiment and by an array of five CFC's in the NPOS experiment. The CFC's were placed inside one of the anode strips, axially displaced from each other by $3 \mathrm{~cm}$ in the MPOS and $1.1 \mathrm{~cm}$ in the NPOS. In the MPOS experiment we also performed measurements of the ion flow toward the cathode. These measurements were done by arrays of six CFC's placed behind holes in the cathode.

In order to evaluate the axially moving ion beam divergence, a multipinhole camera was placed at the short-circuit end of the POS loads. The camera has five holes of $0.05 \mathrm{~cm}$ diameter made in 0.01-cm-thick stainless steel foil. The distance between the holes is $0.7 \mathrm{~cm}$, and the signals were registered on a Polaroid film distanced $0.8 \mathrm{~cm}$ from the holes.

\section{EXPERIMENTAL RESULTS}

\section{A. The NPOS}

1) Axial Ion Flow: During the NPOS opening we have observed an energetic axial flow of ions that propagates toward the short-circuit end of the inductive load. This ion flow was observed for all NPOS conduction times $\tau_{c}=(20-100)$ ns. Typical ion current density signals registered in a single shot by the CFC placed at different azimuthal positions at $\Delta Z=3.5 \mathrm{~cm}$ from the load side of the plasma are shown in Fig. 2(a). An ion beam with $j_{i z} \leq 2.2 \mathrm{kA} / \mathrm{cm}^{2}$ and a full width at half maximum (FWHM) duration of $\tau_{i} \approx 5 \pm 2 \mathrm{~ns}$ was observed at this distance. By placing the radial array of CFC [see Fig. 2(b)] and the axial array of CFC at the same axial position, it was found that the axial ion flow occurs over the entire cross section of the POS interelectrode gap almost simultaneously (within $\pm 3 \mathrm{~ns}$ ). When the magnets on the CFC were removed, an electron beam was observed in front of the ion beam. The electron beam duration was ( $8 \pm 3)$ ns FWHM, and its current density was of the order of tens of $\mathrm{A} / \mathrm{cm}^{2}$.

Fig. 3 shows the axial position of the front of the ion beam relative to the load side of the plasma versus time $(t=0$ is the beginning of $I_{u}$ ). The main axial acceleration occurs in the first few $\mathrm{cm}$ downstream of the load side of the plasma, with the beginning of the NPOS opening. Further in their propagation, the ions are moving with almost constant velocity $V_{i z}=(1.35 \pm 0.25) \times 10^{9} \mathrm{~cm} / \mathrm{s}$. The ion beam duration was observed to be almost constant during its propagation over 30 $\mathrm{cm}$. This, together with the short duration of the beam, allows us to suggest that the ion beam consists of one type of ions that have almost the same energy. Since the NPOS plasma is composed of protons and carbon ions, it is reasonable to suggest that the observed ions are protons.

The energy of the axially moving ions, assuming they are protons, determined by TOF calculations for various $\tau_{\mathrm{op}}$ is shown in Fig. 4(a). One can see that the smaller $\tau_{\text {op }}$ corresponds to higher ion energy. Using $\mathrm{Al}$ foils placed in front of the CFC holes, we observed that the energy of some of the protons (few percent of the total number of protons) exceeds $1.15 \mathrm{MeV}$ but is less than $1.35 \mathrm{MeV}$, which is in agreement with the TOF calculations. For NPOS opening times less than $15 \mathrm{~ns}$, almost all protons have energy $E_{p}>300 \mathrm{keV}$ [see Fig. 4(b)]. The fraction of protons with energy $1.15 \mathrm{MeV} \geq$ $E_{p} \geq 0.75 \mathrm{MeV}$ was $(35 \pm 5) \%$ of the total number of protons. Since the NPOS voltage estimated by $\varphi_{\text {NPOS }} \approx L_{d}\left(d I_{d} / d t\right)$ did not exceed $200 \mathrm{kV}$, one can conclude that the ions are not accelerated solely by the induction voltage. 


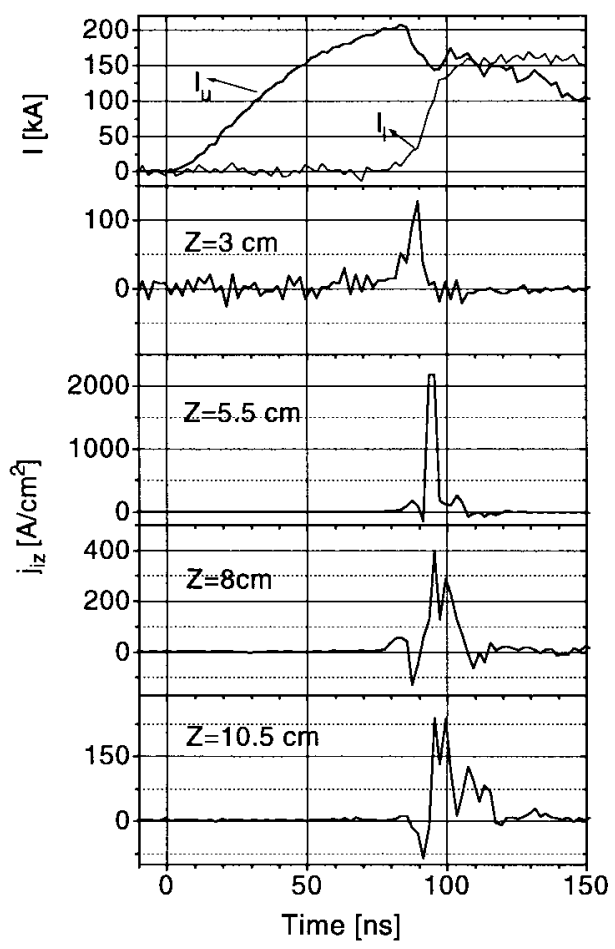

(a)

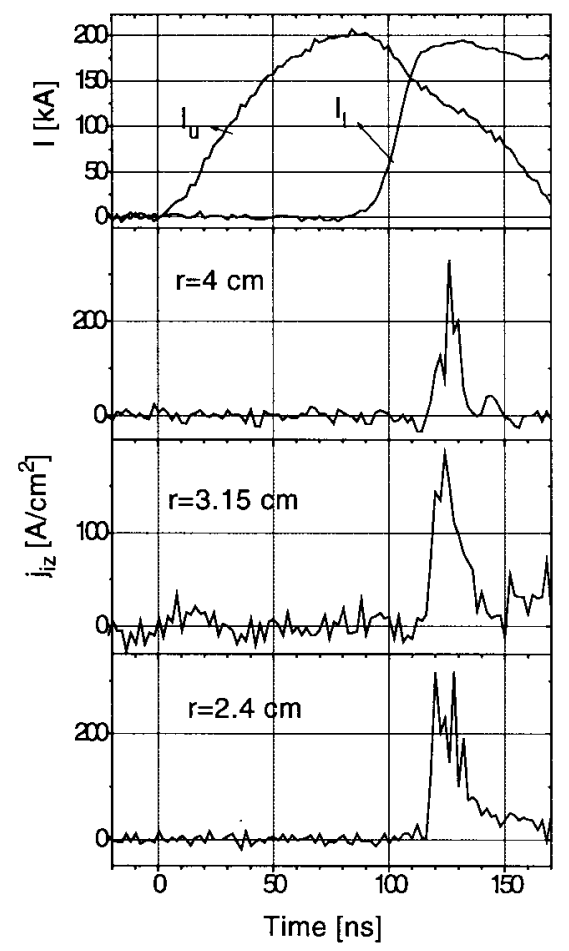

(b)

Fig. 2. (a) The ion current density measured on a single shot by four CFC placed at different axial positions from the load side of the plasma. The upstream $\left(I_{u}\right)$ and the load $\left(I_{d}\right)$ currents of the NPOS are shown above; (b) the ion current density measured is a single shot by three CFC placed at different radial positions at $\Delta Z=12 \mathrm{~cm}$ from the load side of the plasma. The upstream $\left(I_{u}\right)$ and the load $\left(I_{d}\right)$ currents of the NPOS are shown above.

The dependence of the average $j_{i z}$ on axial position for two different $\tau_{c}$ is presented in Fig. 5(a) (the averaging was performed over four CFC's placed at the same axial positions

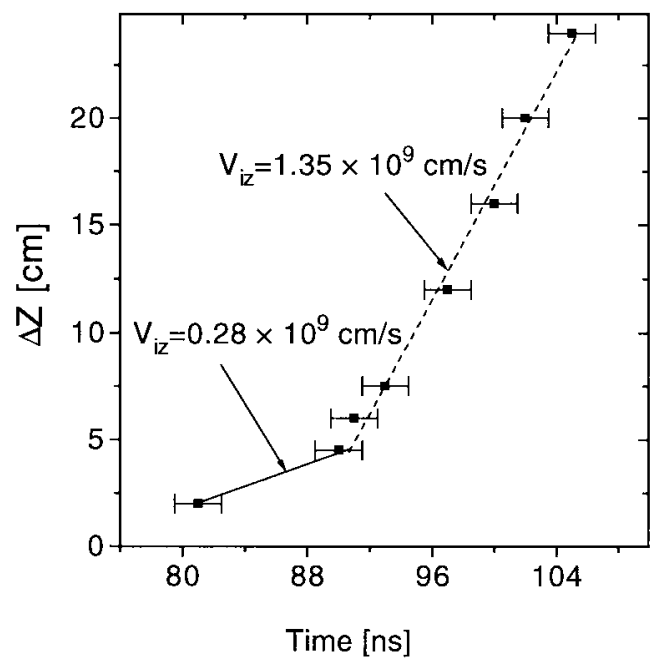

Fig. 3. The axial propagation of the front of the axially moving ion beam for a NPOS conduction time of $70 \pm 5 \mathrm{~ns} . \Delta Z$ is the distance from the load side of the plasma and $t=0$ is the generator current pulse application. The dashed line is a linear fit of the data for $\Delta Z \geq 4 \mathrm{~cm}$.

but at different azimuthal positions). These measurements show a fast decrease of the $j_{i z}$ versus axial distance of propagation that corresponds to an ion beam divergence of $\alpha / 2=17.5^{\circ}$ at $5 \mathrm{~cm} \leq \Delta Z \leq 8 \mathrm{~cm}$ from the load side of the plasma. The average ion current density for various conduction times is presented in Fig. 5(b). It can be seen that $j_{i z}$ is smaller for shorter conduction time. This is presumably because the density of the plasma, which serves a source of ions, is smaller for shorter conduction time.

We can estimate the energy carried by this axially moving ion flow and the total amount of ions. Taking the average ion current density at $\Delta Z=8 \mathrm{~cm}$ from the load side of the plasma to be $j_{i z} \approx 450 \mathrm{~A} / \mathrm{cm}^{2}$, and the ion beam cross section to be $S=51 \mathrm{~cm}^{2}$, one obtains a total ion current of $I_{i z} \approx 23 \mathrm{kA}$. Since $\tau_{i} \approx 5 \mathrm{~ns}$, and taking the average velocity as $1.2 \times 10^{9}$ $\mathrm{cm} / \mathrm{s}$, the proton density in the ion beam is $n_{i z} \approx 2.3 \times 10^{12}$ $\mathrm{cm}^{-3}$, and the total amount of protons is $N_{i z} \approx 10^{15}$. With an average proton energy of $E_{p} \approx 0.75 \mathrm{MeV}$, the energy carried by the ion beam is $W_{i z} \approx 120 \mathrm{~J}$. This ion beam energy corresponds $\approx 10 \%$ of the primary inductively stored energy. Thus, we have observed a powerful high-current ion beam propagating in the axial direction during the NPOS opening.

2) Radial Electron and Ion Flow: The use of the anode array of CFC allowed us to observe the dynamics of radial electron and ion flow toward the anode during the NPOS conduction. Typical signals obtained by this array of CFC are presented in Fig. 6(a). One can see that the electron signals appear almost simultaneously along the entire plasma length. However, the peak of the radial electron current density $j_{e r}$ was observed to propagate axially toward the load side of the plasma. The propagation velocity was observed to be larger for shorter NPOS conduction times. Fig. 6(b) shows the time of the peak radial electron current versus axial position from the plasma generator side for NPOS conduction times of 60 and $125 \mathrm{~ns}$, giving propagation velocities of $\sim 4 \times 10^{8} \mathrm{~cm} / \mathrm{s}$ and $\sim 9$ $\times 10^{7} \mathrm{~cm} / \mathrm{s}$, respectively. The average $j_{e r}$ along most of the 


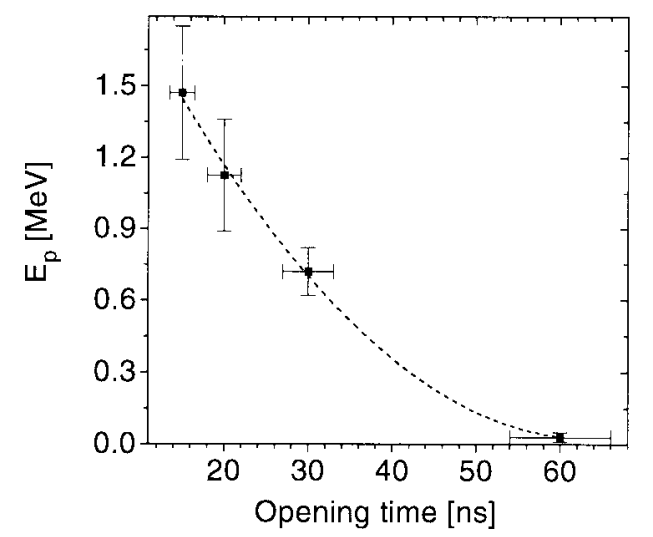

(a)

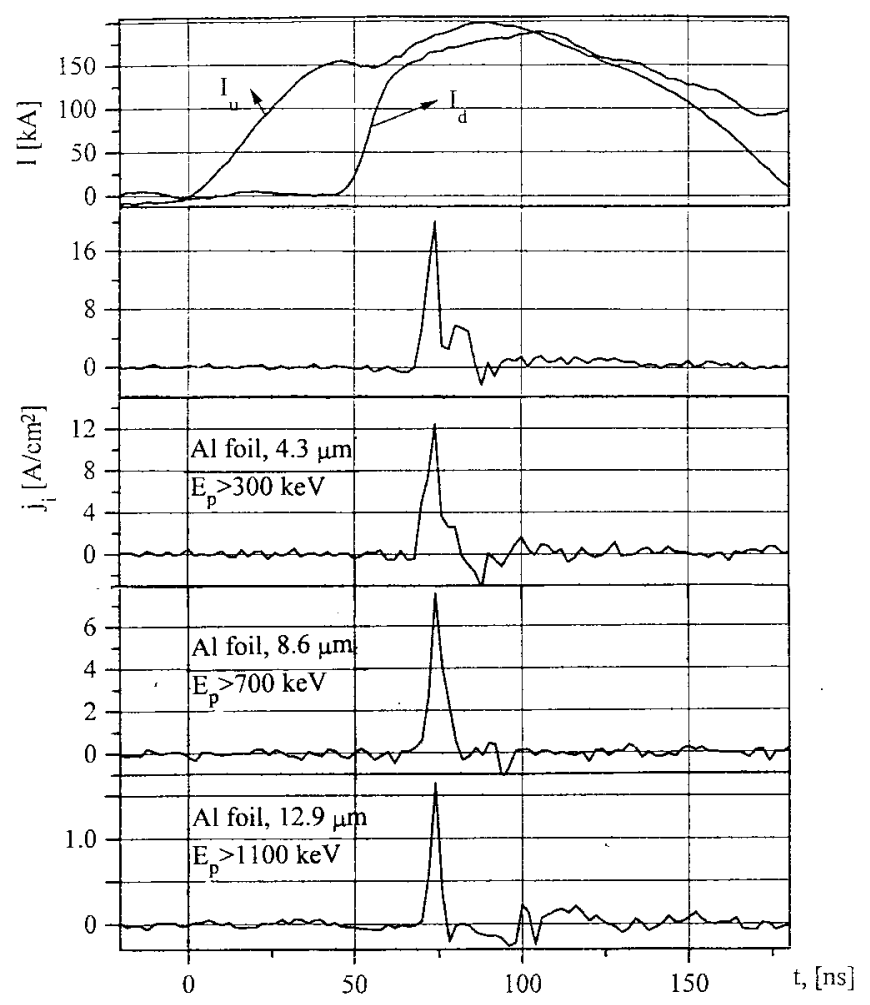

(b)

Fig. 4. (a) Proton energy obtained by TOF calculations versus the NPOS opening time determined at $10-90 \%$ of the maximal load current. (b) Typical ion current density measured by $\mathrm{CFC}$ with $\mathrm{Al}$ foils of different thickness placed in front of collimated hole at $\Delta Z=18 \mathrm{~cm}$ from the load side of the plasma. The upstream $\left(I_{u}\right)$ and the load $\left(I_{d}\right)$ currents of the NPOS are shown above.

plasma length was found to be $j_{e r} \leq 50 \mathrm{~A} / \mathrm{cm}^{2}$ except at the load side of the plasma where $j_{e r}$ increases sharply prior to the NPOS opening. The maximum $j_{e r}$ observed at this position was $j_{e r} \approx(6 \pm 2) \mathrm{kA} / \mathrm{cm}^{2}$. For NPOS conduction time of less than $100 \mathrm{~ns}$, the duration of this electron flow does not exceed $25 \mathrm{~ns}$. To estimate the total radial electron current at its peak, one can take the thickness of this radial electron layer as $d_{l} \approx 1 \mathrm{~cm}$, obtaining $I_{e r}=2 \pi r_{a} d_{l} j_{e r} \approx(160 \pm 30) \mathrm{kA}$ that agrees with the amplitude of the total POS current at this time.

The most distinguished feature of these radial observations was that the electron flow toward the anode was followed by a

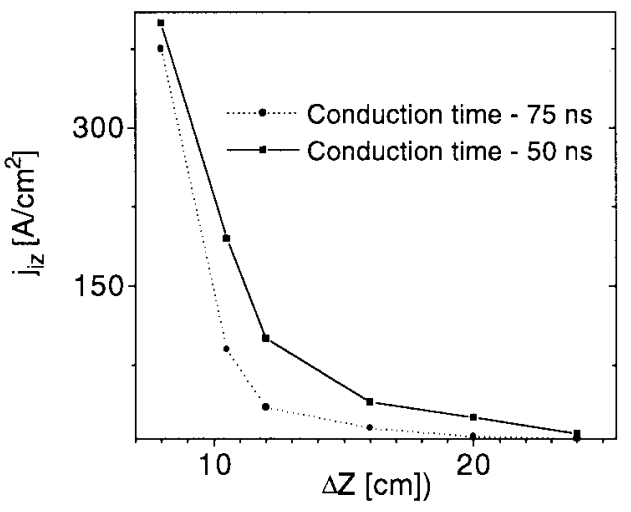

(a)

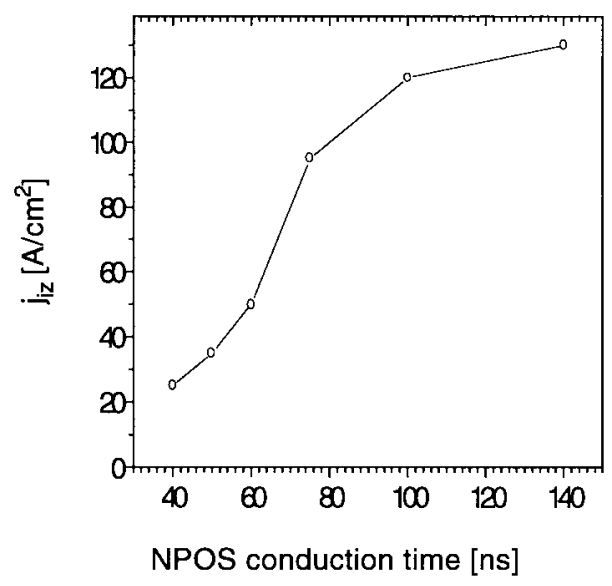

(b)

Fig. 5. The average ion current density for various NPOS regimes: (a) The ion current as a function of distance from the load side of the plasma for conduction times of $50 \mathrm{~ns}$ (dotted line) and $75 \mathrm{~ns}$ (solid line); (b) average ion current density versus NPOS conduction time at an axial distance of $12 \mathrm{~cm}$ from the load side of the plasma.

radial ion flow [see Fig. 6(a)]. The radial ion flow appears first at the generator side of the plasma and propagates toward the load side of the plasma with a velocity of $(1.2 \pm 0.2) \times 10^{8}$ $\mathrm{cm} / \mathrm{s}$. The duration of this radial ion flow was found to be dependent on the axial position and NPOS conduction time. An increase in $\tau_{c}$ or in the axial distance from the generator side of the plasma results in a decrease in the duration of this radial ion pulse. It was also observed that the radial ion current density increases toward the load and has a maximum value up to several hundreds of $\mathrm{A} / \mathrm{cm}^{2}$ at the load side of the NPOS.

We tried to determine the energy of these radial electrons and ions by placing $2 \mu \mathrm{m}$ mylar in front of the CFC holes. However, only the last CFC that was placed at the load side of the plasma has showed an electron signal. Therefore, we can conclude only that the energy of the radially emitted electrons and ions (assuming that they are protons) along the entire plasma, except for its load side, does not exceed 2 and 160 $\mathrm{keV}$, respectively.

\section{B. The MPOS}

1) Axial Ion Flow: Axial flow of fast ions toward the shortcircuit load was observed in the MPOS experiment as well. 


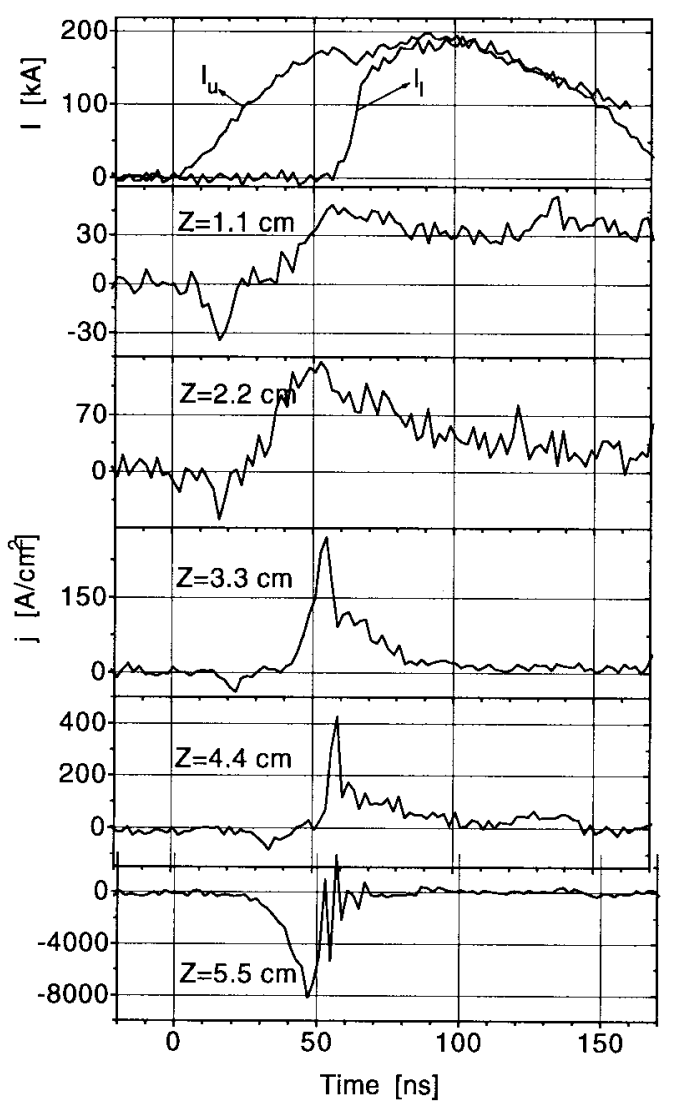

(a)

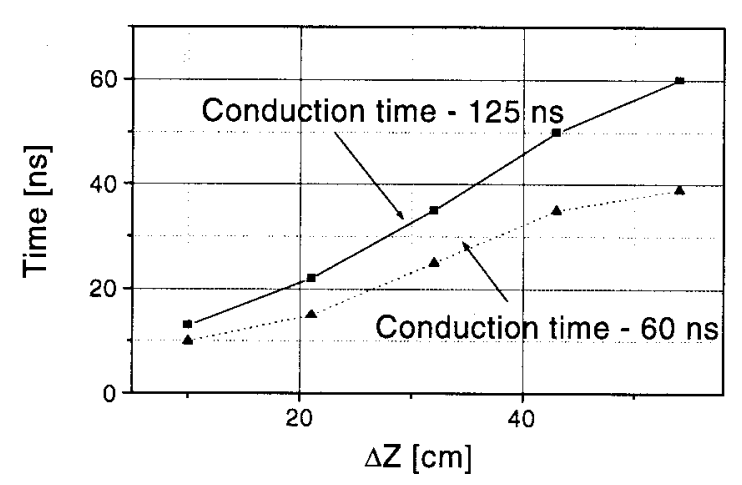

(b)

Fig. 6. (a) Typical current density at different axial positions along the anode during the NPOS operation. $\Delta Z$ is the distance from the generator side of the plasma. (b) Time of the peak of the radial electron current density at different axial positions along the anode for two NPOS conduction times: 60 ns (dotted line) and $125 \mathrm{~ns}$ (solid line).

Typical signals of $j_{i z}$ obtained in a single generator shot by the CFC placed at different axial positions are presented in Fig. 7(a). This ion flow was observed for a wide range of the MPOS conduction times $\tau_{c}=(150-750)$ ns. Results obtained by the axial array of CFC's indicate that the generation of the axial ion flow occurs simultaneously ( $\pm 15 \mathrm{~ns})$ over the entire strip-line cross section. The axial ion flow was observed in the region between the load side of the plasma and the short circuit, even when there was no substantial current in the shortcircuit load except for the current prepulse. By changing the conduction time or the length of the downstream strip line, it

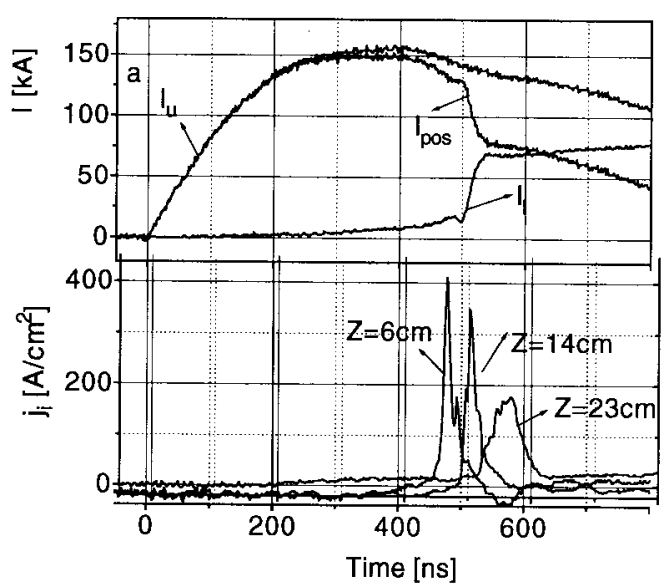

(a)

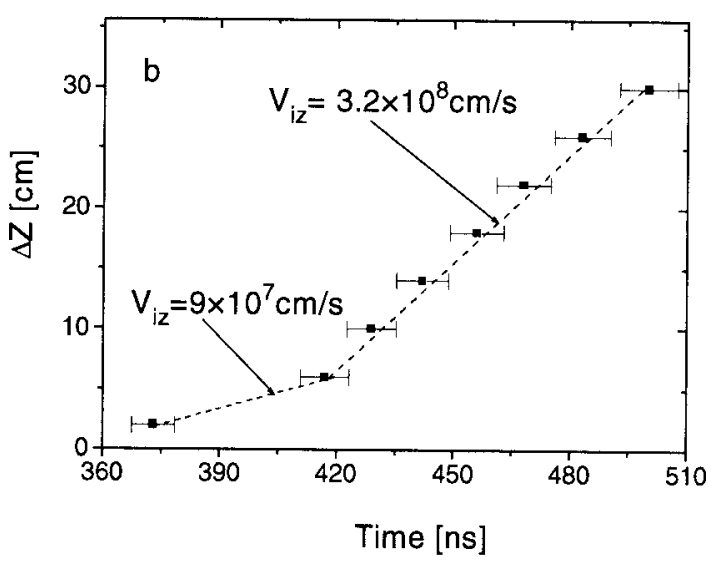

(b)

Fig. 7. (a) The ion beam current density measured at different distances from the load side of the plasma in a single shot. The upstream $\left(I_{u}\right)$, the load $\left(I_{d}\right)$, and the MPOS ( $\left.I_{\mathrm{pos}}\right)$ currents are shown above. (b) The axial distance from the load side of the plasma of the ion beam front versus time (the generator current pulse is applied at $t=0$ ). The POS operation is the same as in (a). The dashed line is a linear fit of the data for $\Delta Z \geq 6 \mathrm{~cm}$.

was verified that the sharp rise of the $I_{d}$ begins only when this axial ion flow reaches the short-circuit load.

The main axial acceleration of ions takes place at the load side of the plasma, similarly to the NPOS case. Fig. 7(b) shows the axial position of the ion beam front versus time where $\Delta Z$ is measured from the load side of the plasma and $t=0$ is the beginning of $I_{u}$. The velocity of the axially moving ions for MPOS conduction times greater than 350 ns was found to be $\approx 3 \times 10^{8} \mathrm{~cm} / \mathrm{s}$, which is three to four times smaller than the ion axial velocity observed in the NPOS experiment. It was observed that the energy of the axially moving ions increases with decreasing $\tau_{c}$, as shown in Fig. 8. As in the NPOS experiment, the ion beam shows only a little spread during its propagation over a distance of $35 \mathrm{~cm}$. It is likely that this ion beam is composed of protons with approximately the same energy. Measurements with the CFC without magnets show that as in the NPOS experiment, the axial ion flow is accompanied by comoving electrons, and that there is a short duration electron beam in the front of the ion flow. 


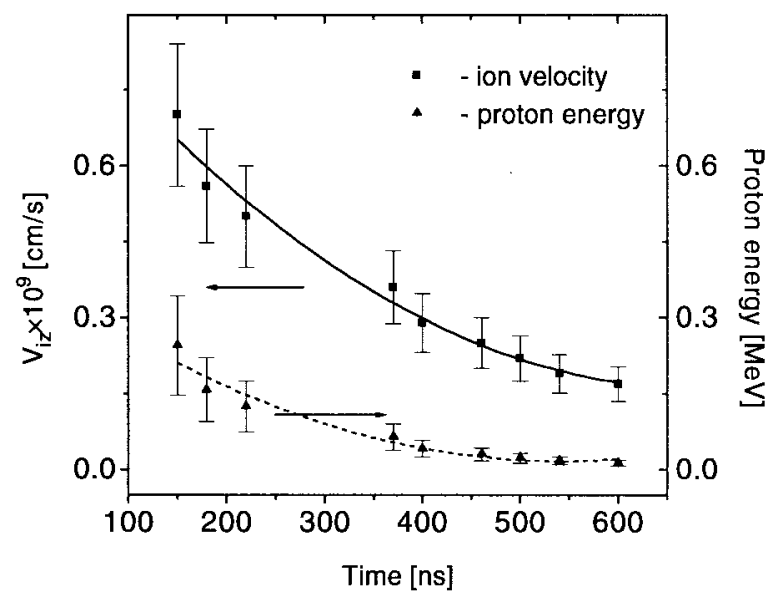

Fig. 8. The ion velocity for $\Delta Z \geq 6 \mathrm{~cm}$ determined by TOF calculations (solid line, left scale) and their energy (assuming protons) (dotted line, right scale) versus MPOS conduction time.

The ion current density and the duration of the axial ion flow at $\Delta Z=(7 \pm 2) \mathrm{cm}$ from the load side of the plasma were found to be up to $1 \mathrm{kA} / \mathrm{cm}^{2}$ and $(30 \pm 10)$ $\mathrm{ns}$, respectively. Taking the ion axial velocity to be $3 \times 10^{8}$ $\mathrm{cm} / \mathrm{s}$ (corresponding to proton energy of $45 \mathrm{keV}, \varphi_{\mathrm{POS}} \leq 40$ $\mathrm{kV}$ ) one obtains the ion density $\sim 2 \times 10^{13} \mathrm{~cm}^{-3}$ and the total amount of axially moving ions $\sim 6 \times 10^{15}$. The energy carried by this axial ion flow reaches $\sim 50 \mathrm{~J}$. We found out that the axial ion flow has a higher current density in the middle of the MPOS along the " $Y$ " and " $X$ " directions (see Fig. 9).

The microdivergence of the ion beam in the " $Y$ " and in the " $X$ " directions measured by the multipinhole camera placed at $\Delta Z=12 \mathrm{~cm}$ from the load side of the plasma was found to be $\alpha_{Y} / 2 \approx 11.5^{\circ} \pm 2.5^{\circ}$ and $\alpha_{X} / 2 \approx 3.6^{\circ} \pm 1.2^{\circ}$, respectively. It was found that $j_{i z}$ decreases by six times over a distance of $15 \mathrm{~cm}$ from the load side of the plasma, which corresponds to ion beam divergence of $\alpha / 2 \approx 5^{\circ}$.

By placing the array of magnetically insulated CFC's inside the MPOS plasma at different axial positions, we obtained well-defined axial ion signals inside the entire plasma, which are presented in Fig. 10(a) for a few axial positions. The TOF calculations presented in Fig. 10(b) show that the axial propagation velocity of the ion current is almost constant over the entire length of the plasma, $V_{i z}=(7 \pm 2) \times 10^{7}$ $\mathrm{cm} / \mathrm{s}$. The peak of the ion current density propagates at $V_{i z}=(3.5 \pm 1.5) \times 10^{7} \mathrm{~cm} / \mathrm{s}$. As mentioned in Section II-C, inside the plasma the velocity determined by TOF calculation can be either of ion velocity or a virtual propagation velocity of an accelerating wave moving through the plasma.

It was observed that $j_{i z}$ and $\tau_{i}$ increase as the ion flow propagates toward the load side of the plasma. For instance, at $\Delta Z=5 \mathrm{~cm}$ from the generator side of the plasma $j_{i z}$ and $\tau_{i}$ were $\approx 100 \mathrm{~A} / \mathrm{cm}^{2}$ and $\approx 30 \mathrm{~ns}$, while at the load side of the plasma $j_{i z} \approx 180 \mathrm{~A} / \mathrm{cm}^{2}$ and $(65 \pm 10) \mathrm{ns}$. At the load side of the plasma, the ion density, assuming they are singly charged and have a velocity of $V_{i z}=3.5 \times 10^{7} \mathrm{~cm} / \mathrm{s}$, is estimated

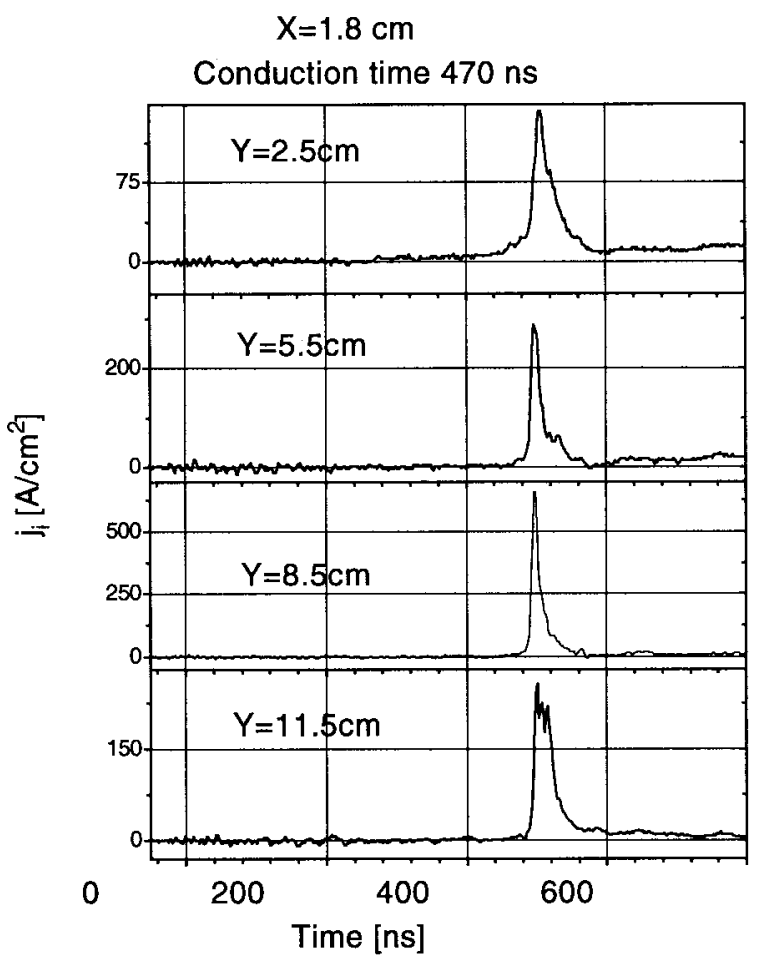

(a)

$Y=8.5 \mathrm{~cm}$

Conduction time 400 ns

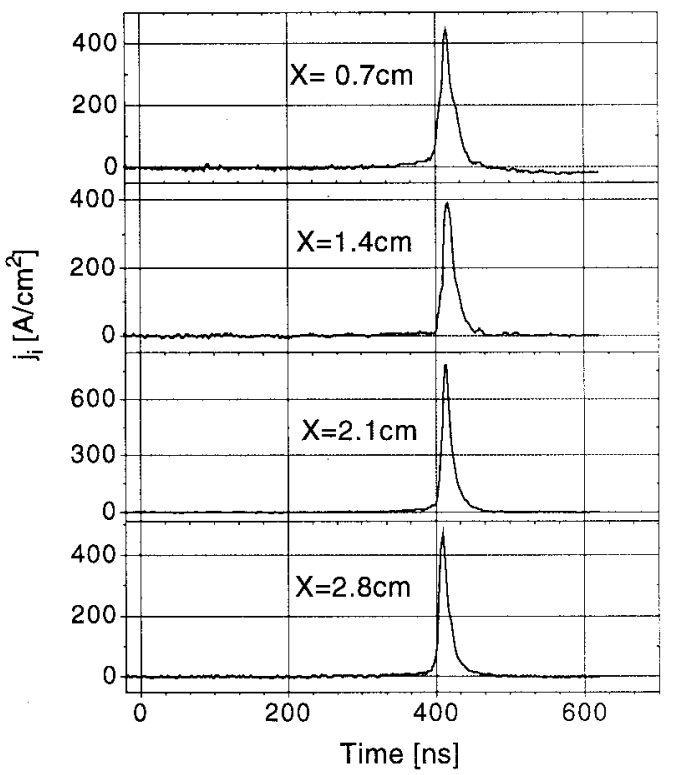

(b)

Fig. 9. Ion current density measured in a single generator shot at $\Delta Z=14$ $\mathrm{cm}$ from the load side of the plasma: (a) different " $Y$ " positions for conduction time of $470 \mathrm{~ns}$; (b) different " $X$ " positions for conduction time of $400 \mathrm{~ns}$.

to be $n_{i} \approx 3 \times 10^{13} \mathrm{~cm}^{-3}$, and the total amount of ions in this flow $N_{i}=(S / e) \int j_{i}(t) d t \approx 3 \times 10^{15}$ (where $S$ is the cross section of the MPOS strip-line interelectrode gap). We would like to note that it is understandable that the reliability of the signals received by the use of the magnetically insulated CFC placed inside the plasma is questionable. Therefore, 


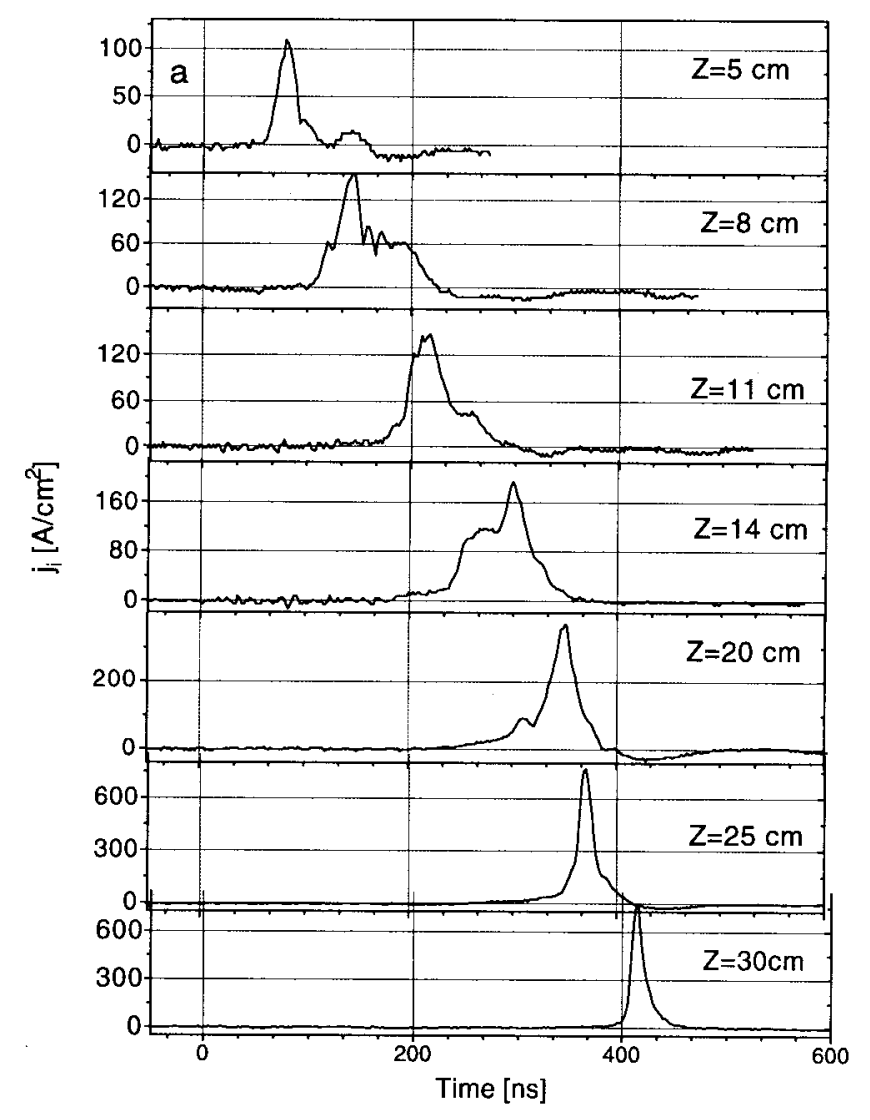

(a)

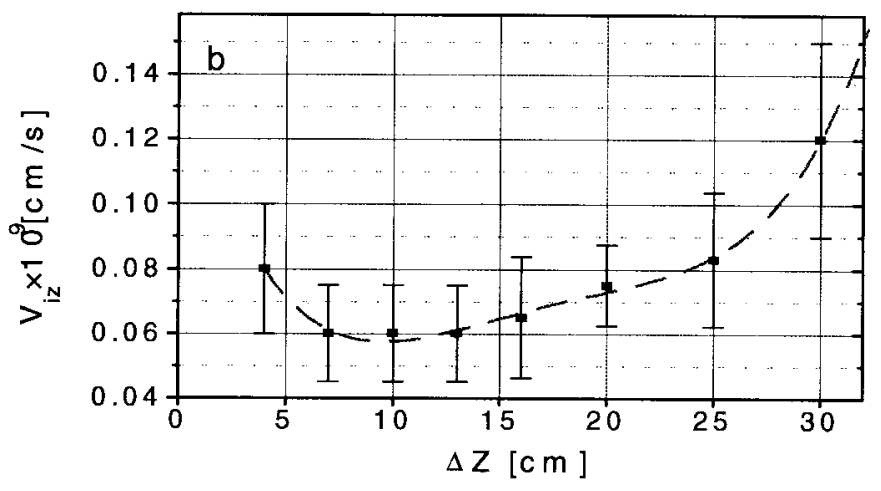

(b)

Fig. 10. (a) The current density of the axially moving ions at different axial positions with respect to the generator side of the plasma. $t=0$ is the beginning of the upstream current. (b) The velocity of the ion front versus axial position determined by TOF calculations.

additional measurements by less ambiguous methods have to be performed in order to prove these quantitative results.

2) Electron and Ion Flow Toward the Electrodes: Measurements by the anode array of the CFC showed results qualitatively similar to the NPOS experiment. Typical signals obtained by this array of CFC are presented in Fig. 11 for $\tau_{c} \approx 450 \mathrm{~ns}$. Early in the current pulse, an almost simultaneous appearance of electron flow toward the anode was observed along the entire length of the plasma. This electron flow has a peak of the current density that propagates in the axial direction with a velocity of $\sim 3 \times$

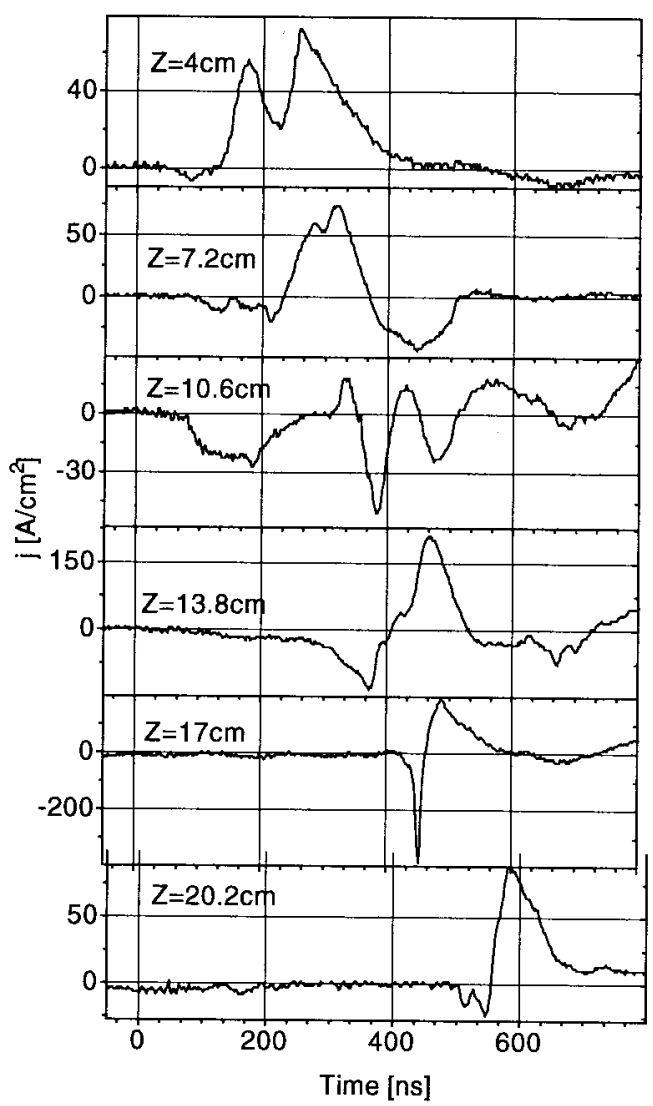

Fig. 11. Electron and ion current density obtained by the anode array of $\mathrm{CFC}$. The MPOS conduction time is $500 \mathrm{~ns}$ and $\Delta Z$ is the distance from the generator side of the plasma.

$10^{7} \mathrm{~cm} / \mathrm{s}$. The peak of the electron current density reaches a few hundreds of $\mathrm{A} / \mathrm{cm}^{2}$ at the load side of the plasma (20 \pm 5 ) ns prior to the beginning of the load current. However, the total electron current amplitude at this place does not exceed $\sim 20 \mathrm{kA}$ (even for a current layer width of $\sim 3 \mathrm{~cm}$ ) that is approximately five times less than the MPOS current amplitude at this time.

Similarly to the NPOS experiment, the electron flow is followed by radial ion flow that appears first at the generator side of the plasma and propagates toward the load side of the plasma. The axial front velocity of this ion-emitting zone is correlated with the velocity of ions axially moving through the plasma. The amplitude of $j_{i z}$ increases toward the load and reaches $\sim 200 \mathrm{~A} / \mathrm{cm}^{2}$ at the load side of the plasma.

The energy of these electrons and ions was found to be less than 2 and $160 \mathrm{keV}$, respectively, because no signals were obtained by the CFC with a $2 \mu$ m mylar in front of the collimating holes. Only at the load side of the plasma have we registered an electron signal that corresponds to electron energy $E_{e} \geq 3 \mathrm{keV}$.

As in the previous research [24]-[27], we have observed an ion flow toward the cathode along the entire plasma length during the MPOS conduction and opening phases. The ion emission starts at the generator side of the plasma within $50 \mathrm{~ns}$ of the application of the upstream current pulse. The 
axial front velocity of the ion emitting zone was found to be $(5 \pm 1.5) \times 10^{7} \mathrm{~cm} / \mathrm{s}$.

\section{DISCUSSION}

The results presented in Section III show similar features for the NPOS and the MPOS experiments. In both experiments we have observed a well-defined, low-energy radial electron and ion flow toward the anode during POS conduction. In both experiments we have observed an energetic ion beam accelerating at the load side of the plasma at a time correlated with POS opening and propagating axially toward the downstream load. This ion beam is accompanied by comoving electrons and has a short duration electron beam at the front of it. These results allow one to suggest that similar physical processes take place during the operation of NPOS and MPOS.

As mentioned in Section I, a fast magnetic field penetration can be explained by EMHD theory. The fastest magnetic field penetration is in a skin layer along the anode [5]. The magnetic field penetrates through the entire plasma as a shock-wave that for coaxial geometry has a velocity [5], [6] $V_{\mathrm{NPOS}}^{p l} \sim(c B / 4 \pi e) r^{2}\left[d\left(1 / n_{e} r^{2}\right) / d r\right]$ where $c$ is the speed of light, $B$ is the magnetic field, $e$ is the electron charge, and $n_{e}$ is the electron density. In the case of weakly collisional plasma, the thickness of the shock front is $\sim c / \omega_{p e}$, where $\omega_{p e}$ is the electron frequency. Fast magnetic field penetration was also predicted by fluid code [19] and particle-in-cell (PIC) code [20], [21] simulations of the POS, which also show a positive floating-potential plasma with respect to both POS electrodes behind the current channel.

However, EMHD theory can be applied to only a certain range of the plasma parameters that satisfy the following inequalities: $V_{A i} \ll j / n_{e} e \ll V_{A e}$ and $c / \omega_{p i} \gg a \gg$ $c / \omega_{p e}$, where $j / n_{e} e$ is the current flow velocity, $V_{A i, e}=$ $B /\left(4 \pi n_{i, e} m_{i, e}\right)^{0.5}$ is the ion and electron Alfven velocities, $\omega_{p i}$ and $\omega_{p e}$ are ion and electron plasma frequencies, respectively, and $a$ is a typical scale of the plasma. It is easy to show that the EMHD approach can not be used for the MPOS case where an magneto-hydrodynamic (MHD) model [22] that includes the Hall effect and takes into account the ion motion has to be used. This approach predicts magnetic field penetration through the plasma $\left(n_{e} \leq 10^{15} \mathrm{~cm}^{-3}\right)$ having density gradients, with velocity $V_{\mathrm{MPOS}}^{p l} \approx c B_{Y} / 8 \pi n_{e} e L_{n}$, where $L_{n}=\left[d\left(\ln n_{e}\right) / d x\right]^{-1}<c / \omega_{p i}$ is the density gradient length scale.

\section{A. Radial Electron Flow}

During both the NPOS and the MPOS conduction there is an electron flow toward the anode that appears almost simultaneously along the entire plasma length, which is consistent with the EMHD model [5]. The peak of the $j_{e r}$ propagates toward the load side of the plasma with velocities $V_{\mathrm{NPOS}}^{\text {pl }} \approx$ $1.6 \times 10^{8} \mathrm{~cm} / \mathrm{s}\left(\tau_{c} \approx 60 \mathrm{~ns}\right)$ and $V_{\mathrm{MPOS}}^{p l} \approx 3 \times 10^{7} \mathrm{~cm} / \mathrm{s}$ $\left(\tau_{c} \approx 450 \mathrm{~ns}\right)$ for the NPOS and MPOS cases, respectively. The velocity of the magnetic field penetration through the entire plasma, which we assume to be associated with the peak of the radial electron flow, can be estimated using the formulas given above. Taking $n_{e} \approx 1 \times 10^{14} \mathrm{~cm}^{-3}, r=4.5$ $\mathrm{cm}, B_{\theta}=0.2 \cdot I_{\mathrm{NPOS}} / r \approx 5 \times 10^{3} \mathrm{G}$ for the NPOS, and $n_{e} \approx 8 \times 10^{14} \mathrm{~cm}^{-3}, L_{n}=1.5 \mathrm{~cm}, B_{Y}=1.2 \cdot j_{\mathrm{NPOS}} \approx 10^{4}$ $\mathrm{G}$ for the MPOS, the calculated velocities are $V_{\text {NPOS }}^{p l} \approx 1 \times 10^{8}$ $\mathrm{cm} / \mathrm{s}$ and $V_{\mathrm{MPOS}}^{p l} \approx 2 \times 10^{7} \mathrm{~cm} / \mathrm{s}$, respectively. These velocities are in satisfactory agreement with the experimentally observed ones.

The observed maximum of the $j_{e r}$ at the load side of the plasma prior to the opening in both the NPOS and MPOS experiments is in qualitative agreement with the results of PIC code simulation [23] and flow impedance model [24]. The latter model predicts large electron losses at the load side of the plasma because of mismatched POS and downstream load-flow impedance.

It can be seen from Figs. 6(a) and 11 that at any instant during the conduction at least two anode CFC's are showing radial electron flow. This indicates that the axial width of the current conduction region is larger than 1 and $3 \mathrm{~cm}$ for the NPOS and MPOS, respectively. These widths are much larger than the shock-front thickness predicted by EMHD or MHD theories, but are in agreement with previous results that have shown broad current channels both in the NPOS and MPOS [3]. The larger thickness of the current layer than predicted by the EMHD model may be related to development of plasma instabilities as suggested in a fluid code simulation [20] and indirectly proved by observation of high frequency electric fields during MPOS [25] and NPOS [26] conduction.

\section{B. Ion Flows}

1) Ion Flow Toward the POS Electrodes: We have shown that the electron flow toward the anode is followed by ion emission in both NPOS and MPOS experiments. In the MPOS experiment, the ion emission region was found to propagate axially along the anode with a velocity that is almost similar to the axial propagation velocity of the ion emission toward the cathode and to the velocity of the ions moving axially inside the plasma. Ions can be emitted toward the POS electrodes due to the Hall field inside the current layer if the electron flow has two-dimensional structure, as predicted by EMHD theory. However, similar ion emission toward the POS electrodes may also occur due to positive potential plasma regions, formed behind the current layer, as predicted by PIC code simulations [20].

The ion flow outside the plasma toward the POS electrodes can redistribute the plasma density during the POS conduction in a way that may enhance the formation of a nonneutral layer during the POS opening. In the NPOS experiment, the average $j_{i a}$ toward the anode was found to be $j_{i a} \approx 150$ $\mathrm{A} / \mathrm{cm}^{2}$ and with the pulse duration being $t_{i a} \approx 30 \mathrm{~ns}$. The average amount of ions leaving the NPOS gap before opening is $N_{i} \approx 2 \pi r_{a} l_{a} t_{i a} j_{i a} / e \approx 5 \times 10^{15}$ where $r_{a}$ is the anode radius $(4.5 \mathrm{~cm})$ and $l_{a}$ is the anode length $(\approx 6$ $\mathrm{cm})$. An estimate of the total amount of ions initially filling the NPOS interelectrode gap gives $N_{i} \approx 6 \times 10^{16}$. Therefore, approximately $8 \%$ of the plasma ions are emitted to the anode. In the MPOS experiment, the decrease in the average plasma density is less and does not exceed a few percent of the initial plasma density. However, if the ion emission toward 
the anode occurs out of a plasma region with dimensions much smaller than the distance between the POS electrodes, the redistribution of plasma density in this particular region before the POS opening may be significantly larger. Additional data concerning the energy and angular distribution of these ions and the region of their acceleration are required in order to clarify the mechanism of their generation.

2) Axial Ion Flow: We have observed, in both the NPOS and the MPOS, the generation of high energy ion fluxes (presumably of protons) toward the load. It was shown that these axial ion fluxes are generated at the load side of the plasma when the POS opening begins. The duration of these ion fluxes is $(5 \pm 2)$ ns and $(30 \pm 10) \mathrm{ns}$ in the NPOS and MPOS, respectively. The ion current density in the vicinity of the load side of the plasma (few $\mathrm{cm}$ downstream) reaches an amplitude of $\geq 1 \mathrm{kA} / \mathrm{cm}^{2}$. In the NPOS the energy of the protons was found to be as high as $1.35 \mathrm{MeV}$. The total energy carried by the axial ion flow reaches 120 and $50 \mathrm{~J}$ for the NPOS and the MPOS cases, respectively. Here it should be mentioned that the same phenomenon of axial ion beam generation during NPOS opening was observed when a planar electron diode was used as the load instead of the short-circuit inductive load.

The phenomenon of a downstream flow of energetic ions is similar to that observed in the early studies of coaxial gaseous plasma guns [28] operated in the so-called "fast" regime, when the initial neutral density did not exceed $10^{15}$ $\mathrm{cm}^{-3}$. Ions with energy up to $80 \mathrm{keV}$ were observed when the supplied voltage amplitude was $\leq 20 \mathrm{kV}$. Several models [29], [30] based on the Hall effect, on thermal expansion of the hot electron component of the plasma, and on plasma instabilities were suggested to explain the appearance of these fast ions. However, in plasma guns, the current channel propagates through gaseous media causing ionization and plasma generation, while in the POS experiments, we have already prepared a highly ionized plasma. Also, the POS conduction times, especially in the NPOS, are much shorter compared with the plasma guns. Therefore, it is not evident that similar physical processes are responsible for the observed ion acceleration.

Recent MPOS experiments on the DECADE generator [31] have shown that there are significant energy losses between the load side of the plasma and the load. Similar results were obtained on ACE-4 generator [32], where the authors have suggested a downstream movement of current carrying dilute plasma in order to explain the observed downstream current temporal behavior. In our experiments, a dilute plasma exists between the main plasma bulk and the load as well, and if the current layer reaches this region, a large Hall electric field would be developed inside it, causing substantial axial ion acceleration [16]. This explanation is in qualitative agreement with the observed axially moving ions and with the absence of a sharp rise of the load current until energetic ion flow reaches the short circuit end of the downstream strip line (see Fig. 7). However, a quantitative comparison requires a detailed knowledge of the density gradient at the plasma load side which is not known in our experiment.
However, it is difficult to use this approach to explain the differences in the observed ion energies of tens of $\mathrm{keV}$ in the MPOS and in the positive polarity central electrode NPOS [16] experiments, and the ion energies of more than $\mathrm{MeV}$ in the present negative polarity NPOS experiment. We suggest that in the NPOS experiment, the axial ion acceleration mechanism is similar to the one observed in "Luce diode" [33] or ion gas dynamics acceleration [34]. In these diode configurations, after the electron beam passes through the anode plasma, the noncompensated space charge of the electron beam creates an electrostatic field that accelerates ions from the boundary of the anode plasma. Fast neutralization of the potential well by the accelerated ions leads to the downstream movement of the potential well and consequently, a fast increase of the distance between its position and the load side of the plasma boundary. This process determines the duration of the ion beam because of a sharp interruption of the ion acceleration at the load side of the plasma. The energy of the axially accelerated ions depends on the energy of the electrons. This explanation is in qualitative agreement with the dependence of the ion energy on the NPOS opening time (see Fig. 3), since the faster the NPOS opening, the higher the NPOS induction voltage, and consequently, the higher the electron energy.

Finally, we think that both acceleration mechanisms, by the Hall field and by collective fields, are active and responsible for the observed axial ion acceleration in the MPOS and the NPOS experiments. However, while in the MPOS experiment the Hall field governs the ion acceleration, in the NPOS experiment, the collective fields are dominant. We note that axial ion acceleration from the load side of the plasma is also predicted by PIC code simulation [23] of the POS.

\section{SUMMARY}

In this paper we have shown that in experiments using a coaxial NPOS (with a negatively charged inner electrode) and a planar geometry MPOS, a high energy, short duration axial flux of ions is generated at the load side of the plasma during POS opening. These high energy ions, probably protons, propagate downstream toward the load, accompanied by comoving electrons. A short duration electron flux was observed in front of the axially moving ions. The ion energy depends strongly on the POS opening duration and may reach the order of MeV. The ion current density depends also on the POS operation and lies in the range of hundreds of $\mathrm{A} / \mathrm{cm}^{2}$. Such ion beams can dissipate a significant amount of the inductively stored energy, resulting in reduced efficiency of energy coupling to a load. The fast ions may also influence the parameters of a load placed in the vicinity of the POS.

In our previous study [16] we suggested, based on spectroscopic measurements of ion velocities, that the ion axial acceleration occurs due to the Hall field at the load side of the plasma. However, it is difficult to use this approach to explain the difference between the ion energies observed in the NPOS and the MPOS. Therefore, we have suggested that in the NPOS, the axial ion acceleration results from collective acceleration by the electric field generated by a downstream electron flow. 
The almost simultaneous appearance at all axial positions of the electron flow toward the anode suggests a fast magnetic field penetration in the vicinity of the anode surface that is consistent with the EMHD model predictions. The EMHD model (for the NPOS) and the MHD model that includes the Hall effect (for the MPOS) also explain the propagation velocity of the peak of the current density of this electron flow that exhibits a maximum at the load side of the plasma and the axial ion motion inside the MPOS plasma. The observed ion emission toward the anode during POS conduction can be explained by either ion acceleration by the Hall field inside the current layer, or due to emission from positive-potential plasma developed behind the current layer.

Additional measurements of the magnetic and electric field distributions in the plasma and the POS downstream region, as well as knowledge of the ion energy distribution, are necessary in order to clarify the validity of the qualitative explanations of the observed results presented here.

\section{ACKNOWLEDGMENT}

The authors are indebted to R. Arad for his contribution in the experiment and fruitful discussions and to Y. Maron, V. M. Bystritskii, L. I. Rudakov, V. Fisher, A. Fruchtman, B. Weber, and J. Greenly for their contribution in discussions and critical comments. The skilled technical assistance of P. Meiri and Y. Maccabi is also acknowledged.

\section{REFERENCES}

[1] B. V. Weber, R. J. Commisso, G. Cooperstein, J. M. Grossmann, D. D. Hinshelwood, D. Mosher, J. M. Neri, P. F. Ottinger, and S. J. Stephanakis, "Plasma erosion opening switch research at NRL," IEEE Trans. Plasma Sci., vol. PS-15, pp. 635-648, 1987.

[2] V. M. Bystritskii, A. A. Kim, B. M. Koval'chuk, Ya. E. Krasik, and G. A. Mesyatz, "Microsecond plasma opening switches," Phys. Particles Nuclei, vol. 23, no. 1, pp. 19-27, 1992.

[3] R. J. Commisso, P. J. Goodrich, J. M. Grossmann, D. D. Hinshelwood, P. F. Ottinger, and B. V. Weber, "Characterization of a microsecond plasma opening switch,” Phys. Fluids, vol. B4, no. 7, pp. 2368-2376, 1992.

[4] B. V. Weber, J. Boller, and R. J. Commisso, "Current distribution in a plasma opening switch," J. Appl. Phys., vol. 45, pp. 1043-1045, 1984

[5] V. M. Bystritskii, Ya. E. Krasik, I. V. Lisitsyn, and A. A. Sinebryukchov, "Experimental investigation of the conduction phase of the microsecond plasma opening switch," IEEE Trans. Plasma Sci., vol. 19, pp. 607-611, 1991.

[6] A. S. Kingsep, K. V. Chukbar, and V. V. Yan'kov, "Electron magnetohydrodynamics," Rev. Plasma Phys., vol. 16, pp. 209-243, 1990.

[7] A. V. Gordeev, A. V. Grechikha, A. V. Gulin, and D. M. Drozdova, "Role of the Hall effect in plasma switches," Sov. J. Plasma Phys., vol. 17, pp. 381-388, 1991

[8] K. Gomberoff and A. Fruchtman, "Fast magnetic field penetration into a cylindrical plasma of a nonuniform density," Phys. Fluids, vol. B5, pp. 2841-2857, 1993.

[9] A. Fruchtman and L. I. Rudakov, "Two-dimensional fast penetration of a magnetic field into a homogeneous plasma," Phys. Rev. Lett., vol. 69, pp. 2070-2073, 1992.

[10] P. F. Ottinger, S. A. Goldstein, and R. A. Meger, "Theoretical modeling of the plasma erosion opening switch for inductive storage applications," J. Appl. Phys., vol. 56, pp. 774-784, 1984.

[11] L. L. Aranchuk, V. M. Babykin, A. S. Chernenko, and A. S. Chuvatin, "Passage of an intense electromagnetic pulse in a vacuum line with a plasma switch," Sov. J. Plasma Phys., vol. 17, no. 5, pp. 317-322, 1991.

[12] P. S. Anan'in, V. M. Bystritskii, V. B. Karpov, Ya. E. Krasik, I. V. Lisitsyn, and A. A. Sinebryukchov, "Electron and ion fluxes during current switching in a microsecond plasma opening switch at $0.2 \mathrm{TW}$," Sov. J. Plasma Phys., vol. 17, no. 1, pp. 39-44, 1991.

[13] B. V. Weber, R. J. Commisso, J. P. Goodrich, J. M. Grossmann, D. D. Hinshelwood, J. C. Kellogg, and P. F. Ottinger, "Investigation of plasma opening switch conduction and opening mechanisms," IEEE Trans. Plasma Sci., vol. 19, pp. 757-766, Oct. 1991.

[14] G. A. Mesyats, A. N. Didenko, E. N. Abdullin, V. M. Bystritskii, A. A. Kim, B. M. Koval'chuk, V. A. Kokshenev, Ya. E. Krasik, and A. A. Sinebryukchov, "Production of intense ion beams in an accelerator with inductive microsecond storage and plasma erosion interrupter," Sov. Phys.-Dokl., vol. 31, no. 7, pp. 557-559, 1986.

[15] G. I. Dolgachev, L. P. Zakatov, and V. A. Skorupin, "Investigation of the plasma opening switch operation in magnetic field," Sov. J. Plasma Phys., vol. 13, pp. 438-440, 1987.

[16] M. Sarfaty, Y. Maron, Ya. E. Krasik, A. Weingarten, R. Arad, R Shpitalnik, A. Fruchtman, and S. Alexiou, "Spectroscopic investigation of the plasma behavior in a plasma opening switch," Phys. Plasmas, vol. 2, no. 6, pp. 2122-2137, 1995.

[17] P. S. Anan'in, V. B. Karpov, Ya. E. Krasik, I. V. Lisitsyn, A. V. Petrov, and V. G. Tolmacheva, "Investigation of plasma opening switches with pulsed plasma guns," Sov. Phys.-Tech. Phys., vol. 36, no. 8, pp. 894-898, 1991

[18] W. Peter and N. Rostoker, "Theory of plasma injection into a magnetic field," Phys. Fluids, vol. 25, no. 4, pp. 730-735, 1982.

[19] R. J. Mason, M. E. Jones, J. M. Grossmann, and P. F. Ottinger, "Magnetic field penetration of erosion-switch plasmas," Phys. Rev. Lett., vol. 61, pp. 1835-1838, 1988.

[20] J. M. Grossmann, R. Kulsrud, J. M. Neri, and P. F. Ottinger, "Investigation of the collisional effects in the plasma erosion opening switch," J. Appl. Phys., vol. 64, no. 12, pp. 6646-6653, 1988.

[21] J. M. Grossmann, P. F. Ottinger, and R. J. Mason, "Current channel migration and magnetic field penetration in a perfectly conducting plasma with emitting, conducting boundaries," J. Appl. Phys., vol. 66, pp. 2307-2314, 1989.

[22] J. D. Huba, J. M. Grossmann, and P. F. Ottinger, "Hall magnetohydrodynamic modeling of a long-conduction-time plasma opening switch," Phys. Plasma, vol. 1, no. 10, pp. 3444-3454, 1994.

[23] S. B. Swanekamp, J. M. Grossmann, P. F. Ottinger, R. J. Commisso, and J. R. Goyer, "Power flow between a plasma opening switch and a load separated by a high-inductance magnetically insulated transmission line," J. Appl. Phys, vol. 76, no. 5, pp. 2648-2656, 1994.

[24] C. W. Mendel, Jr., G. E. Rochau, M. A. Sweeney, D. H. McDaniel, J. P. Quintenz, E. L. Lindman, and J. M. Kindel, "Launched electrons in plasma opening switches," in Int. Workshop Phys. Technol. High Power Opening Switches, Tomsk, Russia, 1989, p. 11.

[25] Yu. P. Golovanov, G. I. Dolgachev, L. P. Zakatov, Yu. G. Kalinin, I. V. Pivinskaya, A. G. Ushakov, and R. V. Chikin, " Investigation of the electric fields in a plasma circuit breaker using the Stark broadening of hydrogen lines," Sov. J. Plasma Phys., vol. 17, no. 7, pp. 466-468, 1991.

[26] S. Alexiou, A. Weingarten, Y. Maron, M. Sarfaty, and Ya. E. Krasik, "Novel spectroscopic method for analysis of nonthermal electric fields in plasmas," Phys. Rev. Lett., vol. 75, no. 17, pp. 3126-3129, 1995.

[27] M. Sarfaty, Ya. E. Krasik, A. Weingarten, A. Fruchtman, and Y Maron, "Energetic ion emission in a positive polarity nanosecond plasma opening switch," in Proc. 11th Int. Conf. High Power Particle Beams, K. Jumgwirth and J. Ullcshmiedt, Eds., vol. 2, Prague, Czech Republic, 1996, pp. 1229-1232.

[28] A. A. Kalmykov, S. A. Trubchaninov, N. A. Naboka, and L. A Zlatopol'skii, "Energy spectra and structure of the plasmoids in a coaxial plasma source," Sov. Phys.-Tech. Phys., vol. 9, no. 6, pp. 779-783, 1964.

[29] N. A. Khizhnyak, "Possible mechanism for the formation of the fast component in an electrodynamically accelerated plasma," Sov. Phys._Tech. Phys., vol. 14, no. 11, pp. 1534-1542, 1970.

[30] V. I. Aref'ev and L. V. Leskov, "Structure of the current front and turbulent acceleration of ions in a pulsed plasma accelerator," Sov. Phys.-Tech. Phys., vol. 17, no. 11, pp. 1822-1828, 1973.

[31] B. V. Weber, P. F. Ottinger, R. J. Commisso, J. R. Goyer, O. Kortbawi, J. Tompson, J. E. Rowley, P. Filios, and M. A. Babineau, "The DECADE performance assessment program," in Proc. 11th Int. Conf. High Power Particle Beams, K. Jumgwirth and J. Ullcshmiedt, Eds., vol. 1, Prague, Czech Republic, 1996, pp. 121-126.

[32] J. Tompson, P. Coleman, C. Gilbert, D. Husovsky, A. R. Miller, J. Rauch, W. Rix, K. Robertson, and E. Waisman, "ACE-4 inductive energy storage power conditioning performance," in Proc. 10th Int. Conf. High Power Particles Beams, vol. 1, San Diego, CA, 1994, pp. 12-16.

[33] V. M. Bystritskii, A. N. Didenko, Ya. E. Krasik, V. S. Lopatin, and V. I. Podkatov, "Collective proton acceleration in a system with an insulate anode," Sov. Tech. Phys. Lett., vol. 4, no. 5, pp. 219-221, 1978.

[34] D. D. Rutov and G. V. Stupakov, "Influence of an ion background on the stored of the electrons in the high-current diode," Sov. J. Plasma Phys., vol. 2, no. 4, pp. 309-315, 1976. 


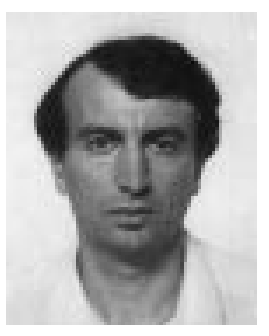

Yakov E. Krasik was born in Ryazan', Russia, in 1953. He received the M.Sc. degree in nuclear physics and the Ph.D. degree in physics and mathematics from Tomsk Polytechnical Institute, Tomsk, Russia, in 1976 and 1980, respectively.

From 1980 to 1991, he was with the Nuclear Research Institute, Tomsk, Russia, as a Senior Research Scientist (until 1987) and as a Head of the High-Power Ion Beam Laboratory (until 1991). From 1991 to 1996, he was a Senior Scientist in the Physics Department, Weizmann Institute of Science, Rehovot, Israel. He is currently a Senior Research Fellow in the Physics Department, Technion, Haifa, Israel. He has worked on high-power electron and ion beam generation, their transport and interaction with various media, and plasma opening switches operated in nanosecond and microsecond time scales.

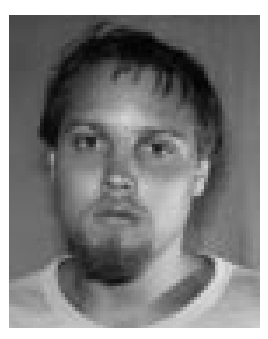

Amit Weingarten was born in Israel in 1965. He received the M.Sc. degree in 1993 from the Weizmann Institute of Science, Rehovot, Israel, where he is pursuing the Ph.D. degree.

$\mathrm{He}$ is currently investigating the opening mechanism of an NPOS at Weizmann. His research is mainly performed using spectroscopic measurements spatially resolved in three dimensions of the magnetic field, electron density, and temperature evolution. 